\title{
Access to Primary Charge Separation Mechanism in Photosynthetic Reaction Centers: Anisotropic Excitation Spectra of Electric Field Modulated Fluorescence Yields
}

\author{
U. Eberl, A. Ogrodnik, and M. E. Michel-Beyerle \\ Institut für Physikalische und Theoretische Chemie, Technische Universität München, \\ Garching, FRG
}

\begin{abstract}
Z. Naturforsch. 45a, 763-770 (1990); received March 17, 1990
Two-step sequential and unistep, superexchange primary electron transfer form primary radical pair states which differ in the direction and magnitude of their dipole moments as revealed in the X-ray structure analysis. The direction can be measured by the excitation anisotropy of electric field induced changes of the fluorescence yield. This method determines angles between the dipole of the primary radical pair and photoselected transition moments (in absorption and emission) of cofactors in the reaction centers. Transitions particularly favourable for discrimination between the two models of primary electron transfer are discussed.
\end{abstract}

Key words: Electric field effect, Anisotropy, Fluorescence, Photosynthesis, Reaction center, Primary charge separation.

\section{Introduction}

Based on the X-ray structure analysis of reaction centers (RCs) of $R$. viridis [1] and $R b$. sphaeroides $[2,3]$, essentially two mechanisms of primary charge separation are discussed. These differ in the role of the monomer bacteriochlorophyll (B) located between the primary donor $\left({ }^{1} \mathrm{P}^{*}\right)$ and a bacteriopheophytin molecule $(\mathrm{H})$. In the two-step mechanism [4-10] according to

$$
{ }^{1} \mathrm{P}^{*} \mathrm{BH} \stackrel{k_{1}}{\longrightarrow} \mathrm{P}^{+} \mathrm{B}^{-} \mathrm{H} \stackrel{k_{2}}{\longrightarrow} \mathrm{P}^{+} \mathrm{BH}^{-},
$$

$\mathrm{P}^{+} \mathbf{B}^{-} \mathbf{H}$ is the first radical pair formed. It develops with a rate constant $k_{1}$ and decays with the rate $k_{2}$ in the subsequent electron transfer (ET) from $\mathbf{P}^{+} \mathbf{B}^{-} \mathbf{H}$ to $\mathrm{P}^{+} \mathrm{BH}^{-}$. In the unistep mechanism the radical ion pair $\mathrm{P}^{+} \mathrm{BH}^{-}$is formed directly from ${ }^{1} \mathrm{P}^{*} \mathrm{BH}$ with the rate $k$ according to

$$
{ }^{1} \mathrm{P}^{*} \mathrm{BH} \stackrel{k}{\longrightarrow} \mathrm{P}^{+} \mathrm{BH}^{-},
$$

where $\mathrm{B}$ mediates superexchange interaction between ${ }^{1} \mathrm{P}^{*} \mathrm{BH}$ and $\mathrm{P}^{+} \mathrm{BH}^{-}[11-23]$. The latter mechanism is favoured by femtosecond absorption spectroscopy at $10 \mathrm{~K}$ [24], whereas the most recent measurements at room temperature support the formation of $\mathrm{B}^{-}$in $\mathrm{RCs}$ of $\mathrm{Rb}$. sphaeroides [25].

Reprint requests to Prof. Dr. M. E. Michel-Beyerle or Dr. A. Ogrodnik, Institut für Physikalische und Theoretische Chemie, Technische Universität München, D-8046 Garching.
An alternative access to the mechanism of the primary electron transfer is by electric field effects on the primary rates $k_{1}$ or $k$ [26-30] which affect the yield of the prompt fluorescence from ${ }^{1} \mathrm{P} * \mathrm{BH}$. Such effects are mainly based on field induced changes in the free energy differences between ${ }^{1} \mathrm{P}^{*}$ and the relevant radical pair states originating essentially from interaction between the local field and the dipole moments $\mu$ of $\mathrm{P}^{+} \mathrm{B}^{-}(\cong 50 \mathrm{D})$ and $\mathrm{P}^{+} \mathrm{H}^{-}(\cong 80 \mathrm{D})$. Those RCs in which $\mu$ is parallel to the local electric field exhibit the largest change of the fluorescence yield. This gives rise to an electric field induced anisotropy of the emission since the angle $\zeta$ between the fluorescence transition moment and the relevant dipole moment $\mu$ of the primary radical pair state is fixed. This elegant method has been developed by Lockhart et al. [31] and applied to RCs of Rb. sphaeroides with the goal to discriminate between the two mechanisms (1) and (2). The angle $\zeta$ measured at $80 \mathrm{~K}$ has been compared with the angles calculated by drawing a vector between the geometric center of $\mathbf{P}$ and the center of either the $\mathbf{B}$ or $\mathrm{H}$ macrocycle using the $\mathrm{X}$-ray structure coordinates for $R$. viridis. As a result, the unistep superexchange mechanism (2) has been suggested to hold at $80 \mathrm{~K}$.

However, the credibility of this result suffers from several uncertainties:

(1) The difference in the angle $\zeta$ between the transition moment of fluorescence and the two moments of $\mathrm{P}^{+} \mathrm{B}^{-} \mathrm{H}$ and $\mathrm{P}^{+} \mathrm{BH}^{-}$is small, $\Delta \zeta \cong 9^{\circ}[31]$. 
(2) The charge distribution at the oxidized dimer $\mathrm{P}^{+}$ may be asymmetric, thus further reducing the difference of the calculated angles to $\Delta \zeta \cong 4^{\circ}$ [32].

(3) For superexchange enhanced ET the electric field dependence of $k$ can be determined by a linear combination of the dipole moments of $\mathrm{P}^{+} \mathrm{B}^{-} \mathrm{H}$ and $\mathrm{P}^{+} \mathrm{BH}^{-}$[33].

(4) The calculated values of $\zeta$ refer to the transition moment of the special pair absorption at $880 \mathrm{~nm}$ [31]. The measured angle $\zeta$, however, is related to the transition moment of emission which is not necessarily identical with the one of absorption. The dichroitic ratio of the fluorescence measured on RCs of $\mathrm{Rb}$. sphaeroides indeed points to such a deviation of the order of $25^{\circ}[34]$.

Due to the uncertainties (1) to (4), the previous set of experiments [31] does not allow unambiguous conclusions on the mechanism, although the dipole moments of $\mathrm{P}^{+} \mathrm{B}^{-} \mathrm{H}$ and $\mathrm{P}^{+} \mathrm{BH}^{-}$are rather different as revealed in the $\mathrm{X}$-ray structure analysis (angle between both $\cong 32^{\circ}$ ). To overcome this undecisive experimental situation, we propose in this paper the measurement of the anisotropy of the electric field effect with respect to the polarization of excitation; i.e. specific transition moments of either B or $\mathrm{H}$ are excited selectively, having angles with the dipole moment which differ more favourable for $\mathrm{P}^{+} \mathrm{B}^{-} \mathrm{H}$ and $\mathrm{P}^{+} \mathrm{BH}^{-}$than the angle $\zeta$ used in [31].

It is the goal of this paper to describe this necessary extension of the previous treatment of electric field induced anisotropy of fluorescence yield [31] and to present specific examples which lead to a large characteristic difference $\Delta \varkappa \cong 30^{\circ}$, where $x$ is the angle between the transition moment of excitation and the dipole moment $\boldsymbol{\mu}$ of the two possible primary radical pairs, $\mathrm{P}^{+} \mathrm{B}^{-} \mathrm{H}$ and $\mathrm{P}^{+} \mathrm{BH}^{-}$. It is self-evident that a large difference is less sensitive to the uncertainties mentioned above.

\section{Analysis of Field-induced Anisotropy of Emission After Photoselective Excitation}

The fluorescence yield $\Phi$ of the primary donor ${ }^{1} \mathrm{P}^{*}$ reflects the competition between the radiative rate $k_{\mathrm{f}}$ and the sum of decay rates including $k$ and $k_{1}$, respectively. For fast primary ET $\left(k, k_{1}>k_{\mathrm{f}}\right)$ as realized in $\mathrm{RCs}, \Phi \cong k_{\mathrm{f}} / k_{\mathrm{ET}}$. This implies that any field induced change of $k_{\mathrm{ET}}$ leads to a concomitant change of the fluorescence yield $\Phi$. For isotropically distributed RCs, the lowest term in the expansion for the field dependence of the fluorescence change $\Delta \Phi=\Phi(E)-\Phi(0)$ is proportional to $(\boldsymbol{\mu} \boldsymbol{E})^{2}$, since any linear contribution cancels due to mirror symmetry with respect to the electric field. This quadratic dependence of $\Delta \Phi$ has been confirmed in experiments $[33,35]$. In the context of this paper the relevant quantity is the anisotropy of the electric field effect rather than its magnitude and field dependence. Therefore, uncertainties with respect to the local strength of the electric field do not affect the reliability of the method as long as the anisotropy of the dielectric properties is negligible.

Polarized excitation of selected transition moments, as e.g. the $Q_{y}$ or $Q_{x}$ transitions of $\mathrm{B}$ or $\mathrm{H}$, photoselects $\mathrm{RCs}$ out of a given isotropic distribution. The probability of excitation of a defined RC is proportional to $\left(p_{\text {ex }} \varepsilon_{\text {ex }}\right)^{2}$ with $p_{\text {ex }}$ denoting the unity vector in the direction of the transition dipole moment and $\varepsilon_{\mathrm{ex}}$ the polarization of excitation. Excitation in the absorption bands of either $\mathrm{B}$ or $\mathrm{H}$ is assumed to populate the donor species ${ }^{1} \mathrm{P}^{*}$ by energy transfer within the selected RC. The following treatment is independent of the mechanism and efficiency of this energy transfer because only the fact of population of ${ }^{1} \mathrm{P}^{*}$ is relevant. The resulting fluorescence yield $\Phi$ from ${ }^{1} \mathrm{P}^{*}$ is proportional to $\left(p_{\mathrm{f}} \varepsilon_{\mathrm{f}}\right)^{2}$ with $p_{\mathrm{f}}$ being the unity vector in the direction of the transition dipole moment of the fluorescence and $\varepsilon_{\mathrm{f}}$ the polarization of the fluorescence. The electric field effect on the fluorescence $\Delta \Phi$ for a RC fixed in space by the internal parameters $\boldsymbol{p}_{\mathrm{ex}}, \boldsymbol{p}_{\mathrm{f}}$, and $\boldsymbol{\mu}$ under a certain configuration of external parameters $\boldsymbol{\varepsilon}_{\mathrm{ex}}, \boldsymbol{\varepsilon}_{\mathrm{f}}$ and $\boldsymbol{E}$ thus is proportional to the product:

$\Delta \Phi\left(\varepsilon_{\mathrm{ex}}, \varepsilon_{\mathrm{f}}, \boldsymbol{E}, \boldsymbol{p}_{\mathrm{ex}}, \boldsymbol{p}_{\mathrm{f}}, \boldsymbol{\mu}\right) \sim\left(\boldsymbol{p}_{\mathrm{ex}} \varepsilon_{\mathrm{ex}}\right)^{2}(\mu \boldsymbol{E})^{2}\left(\boldsymbol{p}_{\mathrm{f}} \varepsilon_{\mathrm{f}}\right)^{2}$.

The overall anisotropy of the electric field effect of an isotropic distribution of RCs with respect to the given external parameters follows from averaging over all possible orientations of the RC, with all the relevant vectors normalized to unity. The magnitude of $\boldsymbol{\mu}$ and $\boldsymbol{E}$ only contributes to the magnitude of the field effect, but not to the anisotropy. The individual factors in $\Delta \Phi$ are not independent from each other, since for each RC we encounter a series of fixed relations between the internal parameters $\boldsymbol{\mu}, \boldsymbol{p}_{\mathrm{ex}}$, and $\boldsymbol{p}_{\mathrm{f}}$. These internal angles are defined as follows:

$$
\begin{aligned}
& \lambda=\operatorname{angle}\left(\boldsymbol{p}_{\mathrm{ex}}, \boldsymbol{p}_{\mathrm{f}}\right), \\
& \chi=\operatorname{angle}\left(\boldsymbol{p}_{\mathrm{ex}}, \boldsymbol{\mu}\right), \\
& \zeta=\operatorname{angle}\left(\boldsymbol{p}_{\mathrm{f}}, \boldsymbol{\mu}\right), \quad 0 \leqq \lambda, \chi, \zeta \leqq 90^{\circ} .
\end{aligned}
$$




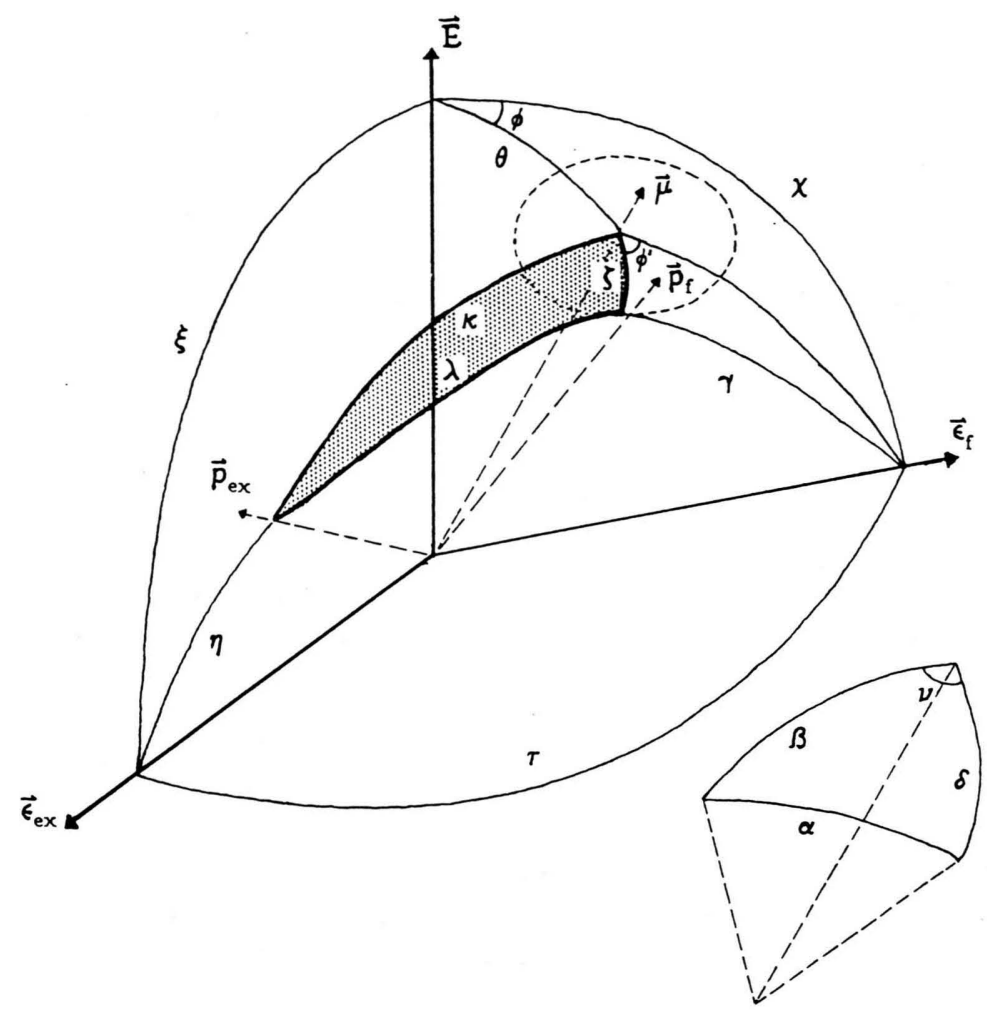

Fig. 1. Geometrical relationship between: the laboratory system - defined by the experimental (unity) vectors of: $E$ (electric field), $\varepsilon_{\mathrm{ex}}$ (polarization of excitation), $\varepsilon_{\mathrm{f}}$ (polarization of fluorescence detection) spanning the external angles $\xi, \chi$, and $\tau$ and the reaction center system - defined by the internal vectors: $\mu$ (the dipole vector of the priradical pair), $p_{\mathrm{f}}$ (the transition dipole of fluorescence) and $p_{\text {ex }}$ (the transition dipole of excitation) spanning the internal angles $\zeta, x$, and $\lambda$. The orientation of the RC-system with respect to the laboratory frame is given by the modified Euler angles $\theta, \phi$, and $\phi^{\prime}$. The experimentally relevant angles $\theta$ (determining the magnitude of the electric field effect), $\eta$ (determining the probability of excitation) and $\gamma$ (determining the probability of fluorescence detection) can be expressed by combinations of the internal, external and Euler angles according to successive application of (5) relating the angle $v$ at one corner of a spherical triangle and the azimutal angles $\alpha, \beta, \delta$ spanning the three sides of the spherical triangle, as illustrated in the insert.
The parameters which can be changed in a specific way are the following ones:

- the excitation wavelength, in order to select different $p_{\text {ex }}$,

- the polarization of absorption and fluorescence, $\varepsilon_{\mathrm{ex}}$ and $\varepsilon_{\mathrm{f}}$,

- the angles $\xi$ and $\chi$ between the electric field $\boldsymbol{E}$ and $\varepsilon_{\text {ex }}, \varepsilon_{\mathrm{f}}$, respectively, and

- the angle $\tau$ between $\varepsilon_{\mathrm{ex}}$ and $\boldsymbol{\varepsilon}_{\mathrm{f}}$.

In the following we adopt the treatment of Reich et al. [36], extending it to the more general problem. In Fig. 1 the basic relations between the various vectors in space and their relative angles are drawn into a segment of the unit sphere. The experimental geometry is defined by the electric field $\boldsymbol{E}$ and the polarizations $\varepsilon_{\mathrm{ex}}$ and $\varepsilon_{\mathrm{f}}$ embracing the angles $\xi, \chi$, and $\tau$. The orientation of an arbitrary RC is given by the modified Euler angles $\theta, \phi$ and $\phi^{\prime}$. The dipole moment $\mu$ is fixed by the angle of latitude $\theta$ and of longitude $\phi$. The possible rotation of the RC around $\mu$ can be characterized by the azimutal angle $\phi^{\prime}$ describing the position of $\boldsymbol{p}_{\mathrm{f}}$ on a cone around $\boldsymbol{\mu}$ with the half-angle of aperture $\zeta$ defined by the internally fixed angle be- tween $\boldsymbol{\mu}$ and $\boldsymbol{p}_{\mathrm{f}}$. The angles $\zeta, \lambda$, and $\varkappa$ define an internally fixed spherical triangle opened by $\boldsymbol{\mu}, \boldsymbol{p}_{\mathrm{f}}$, and $\boldsymbol{p}_{\mathrm{ex}}$. In Fig. 1 these vectors are arranged clockwise in this triangle. Restricting $\lambda, x$, and $\zeta$ to angles $\leqq 90^{\circ}, p_{\mathrm{f}}$ and $\boldsymbol{p}_{\text {ex }}$ might have to be exchanged in the calculation, depending on the actual situation in the RC. The angles

$$
\begin{aligned}
& \theta=\operatorname{angle}(\boldsymbol{\mu}, \boldsymbol{E}), \\
& \gamma=\operatorname{angle}\left(\boldsymbol{p}_{\mathrm{f}}, \boldsymbol{\varepsilon}_{\mathrm{f}}\right), \\
& \eta=\operatorname{angle}\left(\boldsymbol{p}_{\mathrm{ex}}, \boldsymbol{\varepsilon}_{\mathrm{ex}}\right)
\end{aligned}
$$

are the experimentally relevant ones determining (3). The overall anisotropy of the electric field effect, i.e. the average of $\Delta \Phi$ over all possible orientations of RCs, can now be written as

$$
\langle\Delta \Phi\rangle \sim \int_{0}^{\pi} \sin \theta \cos ^{2} \theta \mathrm{d} \theta \int_{0}^{2 \pi} \mathrm{d} \phi \int_{0}^{2 \pi} \cos ^{2} \gamma \cos ^{2} \eta \mathrm{d} \phi^{\prime} .
$$

For the sake of simplicity, the brackets indicating the averaging procedure will be omitted in the following. In order to evaluate the integral, $\gamma$ and $\eta$ have to be expressed as functions of the integrating Euler variables $\theta, \phi$, and $\phi^{\prime}$ and of the internal and external angles defining the laboratory- and RC-system. This is achieved by successive application of the known rela- 
tion between the angles and sides of a spherical triangle, as shown in the insert of Fig. 1:

$$
\cos \alpha=\cos \beta \cos \delta+\sin \beta \sin \delta \cos v .
$$

In general, the integrals in (4) can only be solved numerically.

In the following we will refer to a convenient geometry with orthogonal excitation and detection, which is standard for most fluorescence arrangements as shown in Figure 2. Let the direction of excitation be parallel to the $x$-axis of the laboratory frame $x, y, z$, and let the detection be parallel to the $y$-axis. The electric field is fixed in the diagonal of the $x-y$-plane. The polarization vectors $\varepsilon_{\mathrm{ex}}$ and $\varepsilon_{\mathrm{f}}$ can rotate in the $y-z$ and in the $x-z$ plane, respectively. It is sufficient to measure the four extremal cases, when the polarization vectors are perpendicular to the electric field, i.e. $\boldsymbol{\varepsilon}_{\mathrm{ex}}$ and/or $\boldsymbol{\varepsilon}_{\mathrm{f}}$ are parallel to the $z$-axis, and when they are closest to the electric field axis $\left(45^{\circ}\right)$ in case $\varepsilon_{\text {ex }}$ is parallel to $y$ and/or $\varepsilon_{\mathrm{f}}$ is parallel $x$. We describe these cases by the short notation

$$
\begin{aligned}
& z z: \quad \xi=90^{\circ}, \quad \tau=0^{\circ}, \quad \chi=90^{\circ}, \\
& z x: \xi=90^{\circ}, \tau=90^{\circ}, \chi=45^{\circ}, \\
& y x: \quad \xi=45^{\circ}, \tau=90^{\circ}, \chi=45^{\circ} \text {, } \\
& y z: \quad \xi=45^{\circ}, \tau=90^{\circ}, \quad \chi=90^{\circ},
\end{aligned}
$$

where $z z, z x, y x$, and $y z$ denote the direction of $\boldsymbol{\varepsilon}_{\mathrm{ex}}$ and $\varepsilon_{\mathrm{f}}$, respectively. The angles $\xi, \tau$, and $\chi$ are the angles in the unit sphere in Figure 1.

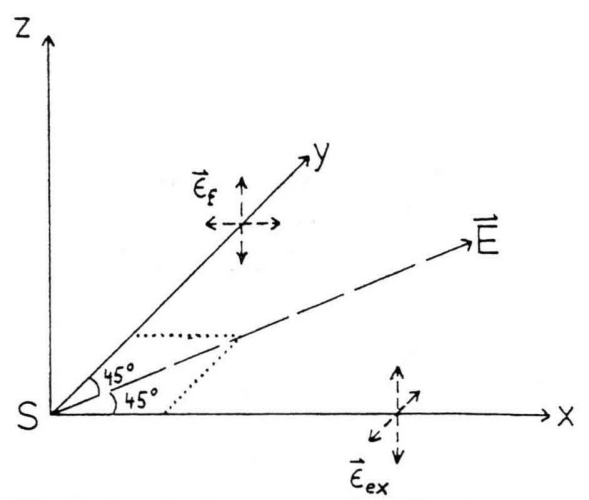

Fig. 2. Standard geometry for a fluorescence experiment with the direction of excitation and detection along the $x$ - and $y$-direction of an orthogonal laboratory frame, respectively. The polarization vectors $\varepsilon_{\mathrm{ex}}$ and $\varepsilon_{\mathrm{f}}$ can be rotated in the $y-z$ and in the $x-z$ plane, respectively. The sample $\mathrm{S}$ is located at the origin with the electric field vector pointing in the direction of the diagonal of the $x-y$ plane.
For this geometry an analytical solution of the integral in (2) can be given in two simple cases:

(I) $\Delta \Phi(z z, \lambda=0) \sim \cos ^{4} \zeta+3 \sin ^{4} \zeta+4 \sin ^{2} \zeta \cos ^{2} \zeta$,

(II) $\Delta \Phi$ (isotropic excitation, $\lambda=0$ )

$$
\sim \cos ^{2} \zeta \cos ^{2} \chi+\frac{1}{3} \sin ^{2} \zeta+\frac{1}{3} \sin ^{2} \chi .
$$

Case (I) may be useful for checking the numerical results of the more general cases.

Case (II) can be approximated by broadband excitation into a couple of differently oriented absorption bands. This is the proposed experimental situation in [31].

In general, the three internal angles $\chi, \zeta, \lambda$ can be determined by numerically fitting the four experimental quantities $\Delta \Phi(z z), \Delta \Phi(z x), \Delta \Phi(y x), \Delta \Phi(y z)$. It is useful to investigate the ratios $\Delta \Phi(z x) / \Delta \Phi(z z)$, $\Delta \Phi(y x) / \Delta \Phi(y z), \Delta \Phi(y x) / \Delta \Phi(z x)$, since they reveal different sensitivity to the different internal parameters. As can be seen in Fig. $3 \mathrm{a}-\mathrm{c}$ the following dependences are valid:

a) The ratio $\Delta \Phi(z x) / \Delta \Phi(z z)$ is particularly sensitive to changes in $\lambda$. This is mainly a consequence of the different probability to detect emission when exciting with orthogonal and parallel polarizations in absence of an electric field. This probability

$$
\frac{\Delta \Phi(z x)}{\Delta \Phi(z z)}=\frac{1+\sin ^{2} \lambda}{1+2 \cos ^{2} \lambda}
$$

increases with increasing $\lambda$, as does the ratio $\Delta \Phi(z x) /$ $\Delta \Phi(z z)$. In practice, $\lambda$ will directly be determined from the measurements with zero external field, being independent of $x$ and $\zeta$.

b) $\Delta \Phi(y x) / \Delta \Phi(y z)$ is particularly sensitive to changes in $\zeta$. This can be rationalized in the following way. While the polarization of excitation $(y)$ is fixed (weak dependence on $\chi$ ), the polarization of emission is $45^{\circ}$ $(x)$ and orthogonal $(z)$ to the electric field direction, respectively. Simultaneously the probability of detection in absence of an electric field is constant, since the polarizations of emission and absorption are orthogonal in both cases (weak dependence on $\lambda$ ).

- If $\zeta \cong 0^{\circ}\left(\boldsymbol{p}_{\mathrm{f}}\right.$ and $\boldsymbol{\mu}$ are parallel), the majority of RCs is selected by the fluorescence polarization to have the dipole moment with an angle of $45^{\circ}(y x)$, and of $90^{\circ}(y z)$ to the electric field, respectively. Thus, $\Delta \Phi(y z)$ exhibits a smaller electric field effect than $\Delta \Phi(y x)$.

- If $\zeta \cong 90^{\circ},(y x)$ selects a rotationally symmetric distribution of dipole moments around the $x$-axis having mainly angles of $45^{\circ} \div 90^{\circ}$ to the electric field. 

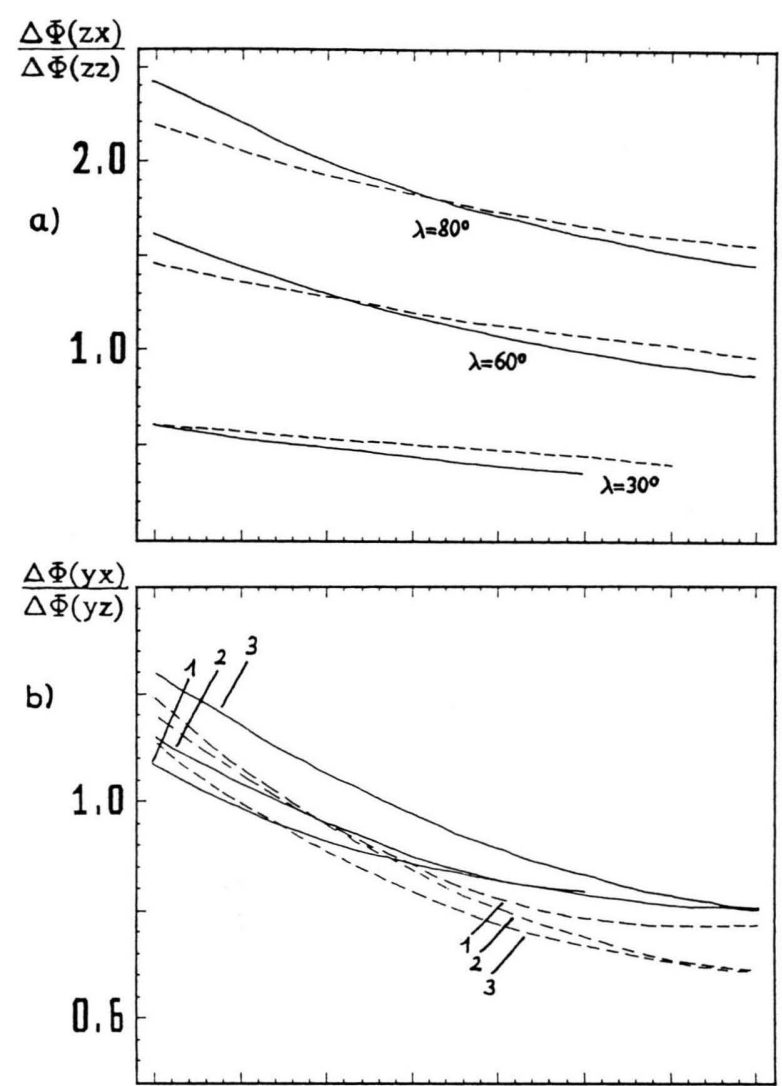

$\Delta \Phi(\mathrm{yx})$

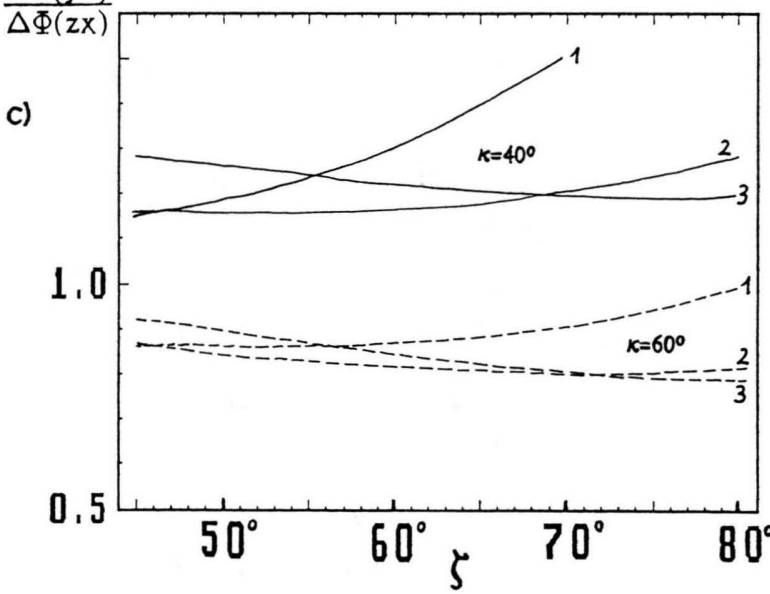

Fig. 3. Plot of the theoretical ratios $\Delta \Phi(z x) / \Delta \Phi(z z)$, a), $\Delta \Phi(y x) / \Delta \Phi(y z), \mathrm{b})$ and $\Delta \Phi(y x) / \Delta \Phi(z x), \mathrm{c})$ versus $\zeta$ with $\lambda$ and $x$ as parameters, according to the numerical solution of (4). $(-): x=40^{\circ},(--): \varkappa=60^{\circ}, 1: \lambda=30^{\circ}, 2: \lambda=60^{\circ}, 3: \lambda=80^{\circ}$. $\zeta$ has been constricted to the region $45^{\circ} \div 80^{\circ}$, which is the relevant one for RCs. $(y z)$ selects a corresponding distribution of the dipole moments around the $z$-axis having angles of $0^{\circ} \div 90^{\circ}$. Now the RCs selected by $(y z)$ will exhibit a larger electric field effect than by $(y x)$.

Summarizing, the ratio $\Delta \Phi(y x) / \Delta \Phi(y z)$ is largest for $\zeta=0$ and smallest for $\zeta=90^{\circ}$, as shown in Figure $3 \mathrm{~b}$.

c) $\Delta \Phi(y z) / \Delta \Phi(z x)$ is particularly sensitive to changes in $\varkappa$. This can be rationalized in analogy to case b), by exchanging excitation and emission.

\section{Overdetermination and Consistency Checks}

Since the difference in $\zeta$ is not sufficient for discrimination between the mechanisms (1) and (2) as argued earlier, differences in $\varkappa$ remain as the most interesting quantity. There are essentially 9 different transitions in the RC, which can more or less be selectively excited, yielding 9 different values for $\lambda$ and $x$ whereas only one value of $\zeta$. Thus, the problem is overdetermined and allows for intrinsic consistency checks:

1) As already mentioned, the angle $\lambda$ between $\boldsymbol{p}_{\mathrm{ex}}$ and $p_{\mathrm{f}}$ can be determined independently from the fluorescence anisotropy in the absence of an electric field.

2) Independent fits of the electric field anisotropy in the different transitions have to yield the same value of the angle $\zeta$ between $\boldsymbol{p}_{\mathrm{f}}$ and $\boldsymbol{\mu}$.

3) The determined set of angles $\chi_{v}, \lambda_{v}$, and $\zeta$ have to be consistent with the relative orientation of the different transition moments (denoted by $v$ ) and the dipole moments extracted from the $\mathrm{X}$-ray structural data.

Thus, the degrees of freedom to fit the electric field anisotropy data are considerably reduced, and the demanded consistency reflects on the accuracy of the experimental data.

\section{Application to the Reaction Center}

Neglecting the dipole moment of the fluorescing state of the special pair, the dipole moment associated with the first ET step in the two-step model for primary charge separation according to reaction (1) originates from $\mathrm{P}^{+} \mathrm{B}^{-} \mathrm{H}$.

In the context of the unistep mechanism (2), ET from ${ }^{1} \mathrm{P}^{*}$ to $\mathrm{P}^{+} \mathrm{BH}^{-}$is mediated by the state $\mathrm{P}^{+} \mathrm{B}^{-} \mathrm{H}$. In this case there are two contributions to the electric field effect on the ET rate, one from the dipole moment of $\mathrm{P}^{+} \mathrm{BH}^{-}$reflecting changes in the Franck-Condon factor (as in the two-step mechanism) and one from the superexchange matrix element. The latter depends in 
$\Delta \Phi(\mathrm{yx})$

$\Delta \Phi(\mathbf{z x})$
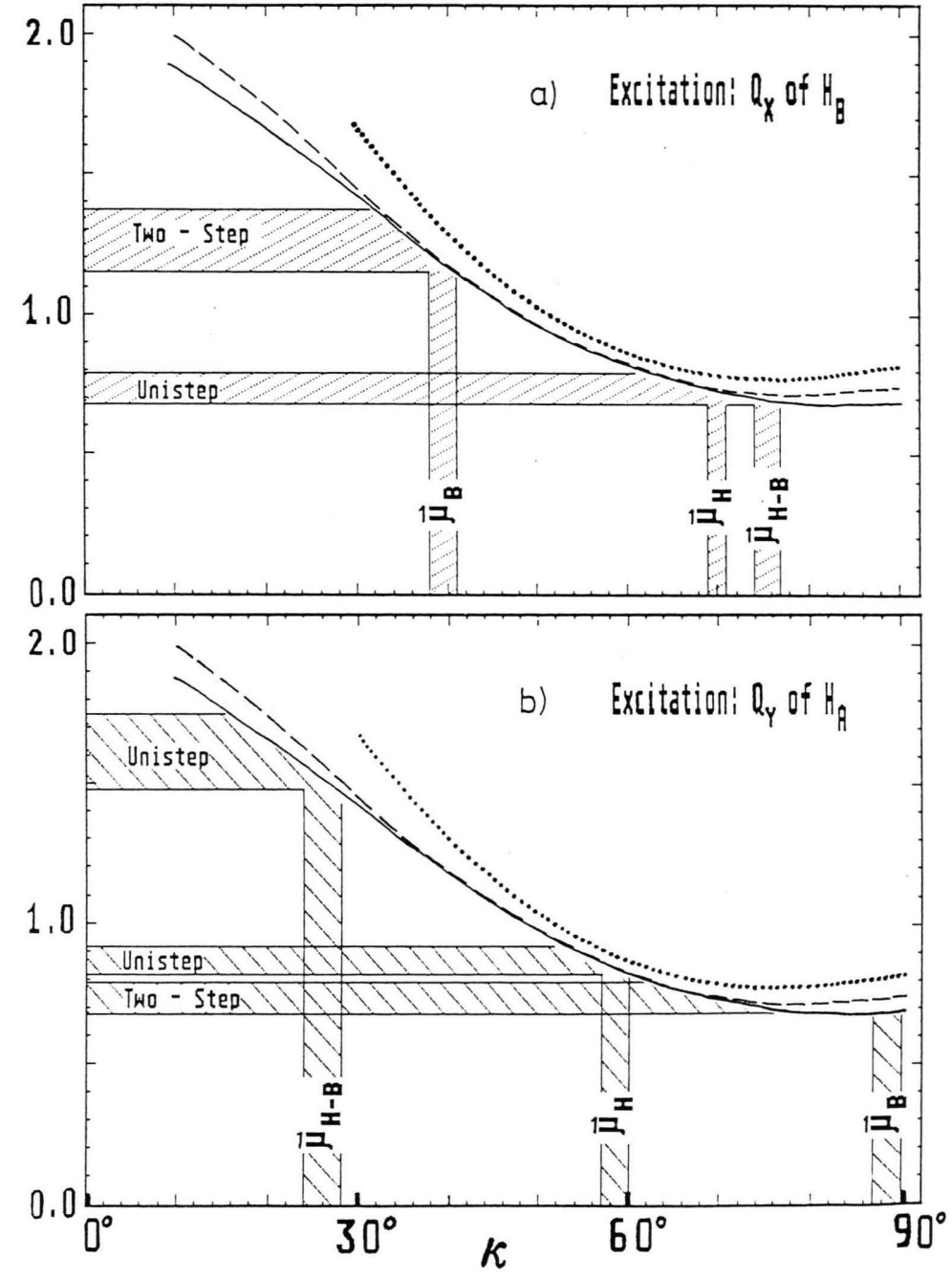

Fig. 4. Both a) and b) show the same plot of $\Delta \Phi(y x) / \Delta \Phi(z x)$ vs. $x$ with $\lambda=30^{\circ}(\cdots)$ $\lambda=50^{\circ}(--), \lambda=70^{\circ}(\longrightarrow)$ as a parameter. $\zeta$ is fixed at $\cong 60^{\circ}$ according to theoretical and experimental values for RCs of $\mathrm{Rb}$. sphaeroides (see text). In a) the angles $x$ between the transition moment of the $Q_{x}$ band of $\mathrm{H}_{\mathrm{B}}$ and the dipole moments $\mu_{\mathrm{B}}$, $\boldsymbol{\mu}_{\mathrm{H}}$, and $\boldsymbol{\mu}_{\mathrm{H}-\mathrm{B}}$ are marked, showing the expected anisotropy after selective excitation of this transition in the frame of the two-step and the unistep mechanism. In b) the corresponding angles $x$ are marked for the $Q_{y}$ transition of $\mathrm{H}_{\mathrm{A}}$. In the case of unistep mechanism one can now distinguish between possible contributions of the Franck-Condon factor (via $\mu_{\mathrm{H}}$ ) and the electronic matrix element (via $\mu_{\mathrm{H}-\mathrm{B}}$ ) to the anisotropy of the electric field effect. a simplified model on a term $\left(\left(\boldsymbol{\mu}_{\mathrm{B}}-\sqrt{E_{\mathrm{r} 12} / E_{\mathrm{r} 13}} \boldsymbol{\mu}_{\mathrm{H}}\right) \cdot \boldsymbol{E}\right)^{2}$ [33]. Here $\mu_{\mathrm{B}}$ and $\mu_{\mathrm{H}}$ are the dipole moments of the radical pair states $\mathrm{P}^{+} \mathrm{B}^{-} \mathrm{H}$ and $\mathrm{P}^{+} \mathrm{BH}^{-}$, respectively (weighted according to their magnitude), while $E_{\mathrm{r} 12}$ and $E_{\mathrm{r} 13}$ are the reorganisation energies which determine the primary rates $k_{1}$ and $k$, respectively. For the sake of simplicity, we define an effective dipole moment $\mu_{\mathrm{H}-\mathrm{B}}$ and assume in the following that $\sqrt{E_{\mathrm{r} 12} / E_{\mathrm{r} 13}} \cong 1$. The contribution from the Franck-Condon factor is given by $\left(\boldsymbol{\mu}_{\mathrm{H}} \boldsymbol{E}\right)^{2}$. The prevalence of either of both terms depends on the vertical energy difference between the transition state and $\mathrm{P}^{+} \mathrm{B}^{-} \mathrm{H}$, on $E_{\mathrm{r}}$ and on the temperature.
From X-ray structural data, an angle between $\mu_{\mathrm{B}}$ and $\mu_{\mathrm{H}}$ of $\cong 32^{\circ}$ and between $\mu_{\mathrm{B}}$ and $\mu_{\mathrm{H}-\mathrm{B}}$ of $\cong 67^{\circ}$ has been calculated [37, 38]. This limits the maximum possible difference $\Delta \varkappa$ discriminating between model (1) and model (2) to $32^{\circ}$ and $67^{\circ}$. Inspection of different absorption bands in the RC of R. viridis revealed that the $Q_{y}$ transition of $\mathrm{H}$ at the active branch (i.e. $\mathrm{H}_{\mathrm{A}}$ ) as well as the $Q_{x}$ transition of $\mathrm{H}$ at the inactive branch (i.e. $\mathrm{H}_{B}$ ) yield favourable values of $x$ for the different combinations of transition and dipole moments (vertical and horizontal row in Table 1) $[37,38]$. For both transitions $\Delta x$ is almost maximal. 
Table 1.

\begin{tabular}{llll}
\hline$\varkappa$ & $\boldsymbol{\mu}_{\mathrm{B}}$ & $\boldsymbol{\mu}_{\mathrm{H}}$ & $\boldsymbol{\mu}_{\mathrm{H}-\mathrm{B}}$ \\
\hline$Q_{y}\left(\mathrm{H}_{\mathrm{A}}\right)$ & $89^{\circ}$ & $58.5^{\circ}$ & $25.5^{\circ}$ \\
$Q_{x}\left(\mathrm{H}_{\mathrm{B}}\right)$ & $39^{\circ}$ & $70^{\circ}$ & $75.5^{\circ}$ \\
\hline
\end{tabular}

In Fig. $4 \mathrm{a}, \mathrm{b}$ the ratio $\Delta \Phi(y x) / \Delta \Phi(z x)$ is shown as a function of $x$ for three different angles $\lambda=30^{\circ}, 50^{\circ}$, and $70^{\circ}$. Whereas, there is only a weak dependence on $\lambda$, the ratio $\Delta \Phi(y x) / \Delta \Phi(z x)$ decreases monotonically in the range $0<x<70^{\circ}$. The fixed value of $\zeta$ has been assumed to be $\cong 60^{\circ}$, in agreement with calculations based on X-ray data [32] and recent measurements of the electric field effect [35], and is close to the value determined by Lockhart et al. [31]. Furthermore, $\Delta \Phi(y x) / \Delta \Phi(z x)$ depends only slightly on $\zeta$, as has been shown in Figure $3 \mathrm{c}$. Measurements of the anisotropy of the electric field effect exciting in the $Q_{x}$ band of $\mathrm{H}_{\mathrm{B}}$ (Fig. $4 \mathrm{a}$ ) must yield a value of $\Delta \Phi(y x) /$ $\Delta \Phi(z x)$ clearly larger than 1 in case $\mu_{\mathrm{B}}$ is involved, whereas a value significantly smaller than 1 is expected for the two other dipole moments in Table 1. The first case indicates the prevalence of the two-step mechanism whereas otherwise the dominance of the unistep mechanism must be concluded, independent of whether $\boldsymbol{\mu}_{\mathrm{H}}$ or $\boldsymbol{\mu}_{\mathrm{H}-\mathrm{B}}$ determine the electric field dependence. By exciting alternatively the $Q_{y}$ band of $\mathrm{H}_{\mathrm{A}}$ (Fig. $4 \mathrm{~b}$ ), a distinction of $\mu_{\mathrm{B}}$ and $\mu_{\mathrm{H}}$ is hardly possible, since $\Delta \Phi(y x) / \Delta \Phi(z x)$ is very flat in this range of $x$. In the case of the superexchange mechanism, however, it is possible to infer whether the electric field mainly affects the Franck-Condon contribution to the rate via $\mu_{\mathrm{H}}\left(\varkappa=58.5^{\circ}\right)$ or the superexchange enhanced electronic coupling via $\mu_{\mathrm{H}-\mathrm{B}}\left(\varkappa=25.5^{\circ}\right)$.

\section{Conclusions}

We have shown that measurements of the excitation anisotropy of the electric field induced fluorescence change at selected transitions, which must be different from the transition of fluorescence, can give full information on the direction of the dipole moment of the primary radical pair in the reaction center. From the analysis, excitation transitions are predicted which are particularly suited for the discrimination between the two possible radical pairs formed in the primary electron transfer process.

This way of measuring and exploiting electric field effects on the polarization of fluorescence constitutes a further development of the method introduced by Lockhart et al. [31]. The extension has become necessary since after isotropic excitation only the projection of the relevant dipole moment on the transition of fluorescence can be determined [31]. In the RC this projection is similar for the two possible radical pairs $\mathrm{P}^{+} \mathrm{B}^{-} \mathrm{H}$ and $\mathrm{P}^{+} \mathrm{BH}^{-}$which can emerge as products of the primary ET process.

However, the final reliability of the experimental results obtained by this method depends on the purity of the analysed fluorescence signal. Although the fluorescence measured is solely due to the emission from dimers, structural and energetic heterogeneities could lead to a slowing down of the primary electron transfer rate. As a result of this, the majority of the steady state fluorescence signal can be due to emissions from a minority of distorted RCs. This source of uncertainty can only be excluded by application of the method proposed in this paper to fluorescence signals resolved in the time domain of primary electron transfer.

The determination of the orientation of the electric dipole moment of the primary radical pair represents an approach to the mechanism of primary charge separation completely different from and complementary to the attempt to identify the primary electron acceptor in time-resolved absorption spectroscopy. It is the beauty of the proposed anisotropic excitation method of electric field induced fluorescence changes that it is still applicable under conditions where mere absorption spectroscopy fails, i.e. when the concentration of the possible intermediate $\mathrm{P}^{+} \mathrm{B}^{-} \mathrm{H}$ is low due to $k_{2} \gg k_{1}$.

\section{Acknowledgements}

We thank P. O. J. Scherer and S. F. Fischer for supplying results concerning the orientation of the various transition moments and Rolf Heckmann for drawing the pictures. We are very grateful to Profs. M. Bixon and J. Jortner, Tel-Aviv, for critically reading the manuscript and stimulating discussions. Financial support by the Deutsche Forschungsgemeinschaft (Sonderforschungsbereich 143) and the Alfried Krupp von Bohlen und Halbach-Stiftung (Eberl) is gratefully acknowledged. 
[1] J. Deisenhofer and H. Michel, EMBO J. 8, 2149 (1989).

[2] H. Komiya, T. O. Yeates, D. C. Rees, J. P. Allen, and G. Feher, Proc. Natl. Acad. Sci. USA 85, 9012 (1988).

[3] D. E. Budil, P. Gast, C.-H. Chang, M. Schiffer, and J. R. Norris, Ann. Rev. Phys. Chem. 38, 561 (1987).

[4] R. A. Marcus, Isr. J. Chem. 28, 205 (1988).

[5] R. Haberkorn, M. E. Michel-Beyerle, and R. A. Marcus, Proc. Natl. Acad. Sci. USA 70, 4185 (1979).

[6] R. A. Marcus, Chem. Phys. Lett. 133, 471 (1987).

[7] S. V. Chekalin, Ya. A. Matveetz, A. Ya. Shkuropatov, V. A. Shuvalov, and A. P. Yartzev, FEBS Lett. 216, 245 (1987).

[8] S. F. Fischer and P. O. J. Scherer, Chem. Phys. 115, 151 (1987).

[9] R. A. Marcus, Chem. Phys. Lett. 146, 13 (1988).

[10] S. Creighton, J.-K. Hwang, A. Warshel, W. W. Parson, and J. Norris, Biochem. 27, 774 (1988).

[11] N. W. Woodbury, M. Becker, D. Middendorf, and W. W. Parson, Biochemistry 24, 7516 (1985).

[12] S. F. Fischer, I. Nussbaum, and P. O. J. Scherer, in: Antennas and Reaction Centers of Photosynthetic Bacteria (M. E. Michel-Beyerle, ed.), pp. 256-263, Springer, Berlin 1985.

[13] J. Jortner and M. E. Michel-Beyerle, in: Antennas and Reaction Centers of Photosynthetic Bacteria (M. E. Michel-Beyerle, ed.), pp. 345-365, Springer, Berlin 1985.

[14] J. Jortner and M. Bixon, in: Protein Structure Moleclar and Electronic Reactivity (R. Austin, E. Buhks, B. Chance, D. DeVault, P. L. Dutton, H. Frauenfelder, and V. I. Gol'danskii, eds.), pp. 277-308, Springer-Verlag, New York 1987.

[15] A. Ogrodnik, N. Remy-Richter, M. E. Michel-Beyerle, and R. Feick, Chem. Phys. Lett. 135, 576 (1987).

[16] J. R. Norris, D. E. Budil, D. M. Tiede, J. Tang, S. V. Kolaczkowski, C. H. Chang, and M. Schiffer, in: Progress in Photosynthetic Research (J. Biggens, ed.) I, pp. 1.4.363-1.4.369, Martinus Nijhoff, Dordrecht 1987.

[17] M. E. Michel-Beyerle, M. Plato, J. Deisenhofer, H. Michel, M. Bixon, and J. Jortner, Biochim. Biophys. Acta 932, 52 (1988).

[18] M. Bixon, J. Jortner, M. Plato, and M. E. MichelBeyerle, in: The Photosynthetic Bacterial Reaction Center. Structure and Dynamics (J. Breton and A. Vermeglio, eds.), pp. 399-420, NATO ASI Series, Plenum, New York 1988.

[19] M. Plato, K. Möbius, M. E. Michel-Beyerle, M. Bixon, and J. Jortner, J. Amer. Chem. Soc. 110, 7279 (1988).

[20] M. E. Michel-Beyerle, M. Bixon, and J. Jortner, Chem. Phys. Lett. 151, 188 (1988).

[21] M. Bixon, M. E. Michel-Beyerle, and J. Jortner, Isr. J. Chem. 28, 155 (1988).

[22] M. Bixon, J. Jortner, M. E. Michel-Beyerle, and A. Ogrodnik, Biochim. Biophys. Acta 977, 273 (1989).

[23] R. A. Friesner and Y. Won, Biochim. Biophys. Acta 977, 99 (1989)

[24] J.-L. Martin, J. Breton, A. J. Hoff, A. Migus, and A. Antonetti, Proc. Natl. Acad. Sci. USA 83, 957 (1986)

[25] W. Holzapfel, U. Finkele, W. Kaiser, D. Oesterhelt, H. Scherer, H. U. Stilz, and W. Zinth, Chem. Phys. Lett. 160, 1 (1989).

[26] Z. D. Popovic, G. J. Kovacs, P. S. Vincett, G. Alegria, and P. L. Dutton, Chem. Phys. 110, 227 (1986).

[27] A. Gopher, Y. Blatt, M. Schönfeld, M. Y. Okamura, G. Feher, and M. Montal, Biophys. J. 48, 311 (1985).

[28] G. Feher, T. Arno, and M. Y. Okamura, in: The Photosynthetic Reaction Center (J. Breton and A. Vermeglio, eds.), NATO ASI Series A 149, pp. 271-287, Plenum Press, New York 1988

[29] D. J. Lockhart and S. G. Boxer, Chem. Phys. Lett. 144, 243 (1988).

[30] Z. D. Popovic, G. J. Kovacs, P. S. Vincett, G. Alegria, and P. L. Dutton, Biochem. Biophys. Acta 851, 38 (1986).

[31] D. J. Lockhart, R. F. Goldstein, and S. G. Boxer, J. Chem. Phys. 89, 1408 (1988).

[32] A. Ogrodnik and M. E. Michel-Beyerle, Z. Naturforsch. $44 \mathrm{a}, 763$ (1989)

[33] S. G. Boxer, R. A. Goldstein, D. J. Lockhart, T. R. Middendorf, and L. Takiff, J. Phys. Chem. 93, 8280 (1989).

[34] U. Eberl et al., to be published.

[35] U. Eberl et al., unpublished results.

[36] R. Reich and S. Schmidt, Ber. Bunsenges. 76, 589 (1972)

[37] M. Plato, W. Lubitz, F. Lendzian, and K. Möbius, Isr. J. Chem. 28, 109 (1988).

[38] P. O. J. Scherer and S. F. Fischer, in: Perspectives in Photosynthesis (J. Jortner and B. Pullman, eds.), p. 361 370, Kluwer Academic Publ. (1990). 